\title{
microRNA-101 inhibits lung cancer invasion through the regulation of enhancer of zeste homolog 2
}

\author{
HYUN MIN CHO ${ }^{1}$, HYO SUNG JEON ${ }^{2}$, SOO YOUNG LEE ${ }^{3}$, KANG JIN JEONG ${ }^{4}$, SOON-YOUNG PARK ${ }^{4}$, \\ HOI YOUNG LEE ${ }^{3,4}$, JUNG UEE LEE ${ }^{5}$, JI HYE KIM ${ }^{6}$, SUN JUNG KWON ${ }^{6}$, EUGENE CHOI ${ }^{6}$, \\ MOON JUN NA ${ }^{6}$, JAEKU KANG ${ }^{4}$ and JI WOONG SON ${ }^{3,6}$
}

\author{
${ }^{1}$ Department of Thoracic Surgery, Konyang University Hospital, Daejeon 302-718; ${ }^{2}$ Department of Biochemistry, \\ School of Medicine, Kyungpook National University, Daegu 700-422; ${ }^{3}$ Myunggok Research Institute for Medical Science, \\ Konyang University, Daejeon 302-718; ${ }^{4}$ Department of Pharmacology, College of Medicine, Konyang University, \\ Daejeon 302-718; ${ }^{5}$ Department of Hospital Pathology, The Catholic University of Korea, Daejeon St. Mary's Hospital, \\ Daejeon 301-723; ${ }^{6}$ Department of Internal Medicine, Konyang University Hospital, Daejeon 302-718, Republic of Korea
}

Received April 12, 2011; Accepted June 9, 2011

DOI: $10.3892 / \mathrm{etm} .2011 .284$

\begin{abstract}
RNAs (miRNAs) may function as oncogenes or tumor-suppressor genes depending on the targets that are regulated. Enhancer of zeste homolog $2(\mathrm{EZH} 2)$ is the target of miR-101 and member of the polycomb repressive complex 2 , which is involved in the methylation of histone $\mathrm{H} 3$ at lysine 27 (H3K27). Therefore, we aimed to ascertain whether or not the overexpression of miR-101 inhibits invasion of lung cancer through regulation of EZH2. In this study, the expression of miR-101 was down-regulated and the expression of EZH2 was up-regulated in lung cancer. Global methylation of H3K27 was higher in metastatic lung cancer than in early lung cancer lesions. Overexpression of miR-101 induced a marked reduction in EZH2 mRNA levels in several lung cancer cell lines. A reduction in the trimethyl $\mathrm{H} 3 \mathrm{~K} 27$ histone mark was detected at the CDH1 promoter in miR-101 precursor-transfected cells. Moreover, the expression of CDH1 and MMP-2 was reversed by miR-101 transfection. Therefore, the overexpression of miR-101 inhibits the invasiveness of lung cancer. miR-101 may be a potent tumor suppressor by altering chromatin structure through repression of EZH2 and may be a potential therapeutic tool for patients with lung cancer.
\end{abstract}

Correspondence to: Dr Ji Woong Son, Department of Internal Medicine, Konyang University Hospital, Daejeon 302-718, Republic of Korea

E-mail: sk1609@hanmail.net

Dr Jaeku Kang, Department of Pharmacology, College of Medicine, Konyang University, Daejeon 302-718, Republic of Korea

E-mail: jaeku@konyang.ac.kr

Key words: microRNA, miR-101, enhancer of zeste homolog 2, invasion, lung cancer

\section{Introduction}

microRNAs (miRNAs) are non-coding, single-stranded RNAs that repress gene expression by interacting with messenger RNA (mRNA) by inhibiting mRNA translation or by inducing mRNA cleavage (1-3). To date, more than 600 human miRNAs have been identified and up to $30 \%$ of all protein-coding genes are estimated to be regulated by miRNAs $(4,5)$. Although the precise function of individual miRNAs has not been characterized, biochemical and genetic studies have revealed that miRNAs regulate a variety of biological processes, such as cell proliferation, apoptosis, development and differentiation. In addition, it has been reported that certain miRNAs are aberrantly expressed in human cancers, suggesting that miRNAs may function as oncogenes or tumor-suppressor genes depending on the targets that are regulated (6-9).

A subgroup of miRNAs control promoter methylation and chromatin structure by regulating DNA methyltransferases and histone modifiers. Enhancer of zeste homolog 2 (EZH2) is a member of the polycomb repressive complex 2 , which is involved in the methylation of histone $\mathrm{H} 3$ at lysine 27 (H3K27). EZH2 contributes to the epigenetic silencing of target genes and regulates the biology of cancer cells. Overexpression of miR-101 inhibits EZH 2 and has a strong influence on the phenotype of various cancers, including prostate, breast cancer and bladder transitional cell carcinoma. In addition, EZH2 has been identified as a target of miR-101 by the luciferase reporter assay $(10,11)$.

In the present study, the differential expression of miR-101 and EZH2 in lung cancer and normal lung tissues was determined. We hypothesized that miR-101 has a role in lung cancer invasion. To test this hypothesis, a cell invasion assay was performed, and the expression of genes related to cancer invasion was determined.

\section{Materials and methods}

Cell lines. Lung cancer cells (A549, H522 and HCC1438) were maintained in RPMI-1640 medium (Gibco BRL, Rockville, 
MD, USA) with $10 \%$ FBS and antibiotics (100 U/ml of penicillin and $100 \mathrm{mg} / \mathrm{ml}$ of streptomycin).

Tissues. Tumor and corresponding normal lung tissue specimens were obtained from Korean patients with non-small cell lung cancer who underwent curative resection at the Konyang University Hospital. None of the patients had received chemotherapy or radiotherapy prior to surgery. Informed consent was obtained from each patient before surgery. This study was approved by the Bioethics Committee of Konyang University Hospital. All of the tumor and macroscopically normal lung tissue samples were obtained at the time of surgery, and were rapidly frozen in liquid nitrogen and stored at $-80^{\circ} \mathrm{C}$ until analysis. Tissue samples were histologically confirmed by H\&E staining.

Immunohistochemistry (IHC) of the global methylation of H3K27 (H3K27me). Sections $(4 \mu \mathrm{m})$ of formalin-fixed paraffin-embedded tissues were deparaffinized. The antigen was retrieved with $0.01 \mathrm{M}$ citrate buffer $(\mathrm{pH}$ 6.0) by heating the sample in an autoclave at a controlled final temperature of $121^{\circ} \mathrm{C}$ for $30 \mathrm{~min}$. Endogenous peroxidase activity was blocked using $0.3 \%$ hydrogen peroxide. For immunohistochemical detection of $\mathrm{H} 3 \mathrm{~K} 27 \mathrm{me}$, specimens were incubated for $1 \mathrm{~h}$ at room temperature with the histone $\mathrm{H} 3$ (trimethyl K27) antibody (1:100; Abcam, Cambridge, MA, USA). The sections were visualized with 3-3'-diaminobenzidine (DAB), and tissue sections were counterstained with Mayer's hematoxylin. IHC of H3K27me was scored as follows: 0, no staining; 1, weak or focal staining of the cancer cell nuclei; 2 , moderate nuclear staining in most cancer cells; and 3, strong nuclear staining in most cancer cells. When the score was $\geq 1$, the section was considered to have positive H3K27me expression.

miRNA precursor transfection. Cells were plated in 6-well plates at a density of $1.5 \times 10^{5}$ cells/well. The next day, cells were transfected with $50 \mathrm{nM}$ Pre-miR ${ }^{\mathrm{TM}}$ miRNA Precursor (Ambion Inc., Austin, TX, USA) and Pre-miR ${ }^{\mathrm{TM}}$ miRNA Precursor-Negative Control \#1 (Ambion Inc.) with Oligofectamine (Invitrogen, Carlsbad, CA, USA) according to the manufacturer's instructions.

Quantitative real-time PCR assay. Total RNA was isolated with TRIzol (Gibco-BRL, Glasgow, UK) according to the protocols of the manufacturer, and the first strand of cDNA was synthesized using the oligo (dT) primer system (SuperScript III First-Strand Synthesis System; Invitrogen). Aliquots of the reaction mixture were used for the qPCR amplification with the IQ5 system (Bio-Rad Laboratories, Hercules, CA, USA) using iQ SYBR Green Supermix (Bio-Rad). The PCR was run for 40 cycles of denaturation at $95^{\circ} \mathrm{C}$ for $15 \mathrm{sec}$, annealing at $55^{\circ} \mathrm{C}$ for $15 \mathrm{sec}$ and elongation at $72^{\circ} \mathrm{C}$ for $15 \mathrm{sec}$. Gene expression was quantified by the comparative $\mathrm{CT}$ method, by normalizing CT values to the housekeeping gene $\beta$-actin. After amplification, melting curve analysis was performed to ensure the specificity of the products.

TaqMan miRNA expression assay. qRT-PCR analysis for miRNAs was performed in duplicate with a TaqMan MicroRNA assay kit (Applied Biosystems, Foster City, CA,
USA) according to the manufacturer's instructions, and RNU6B was used for normalization.

Western blot analysis. Cells were lysed in Pro-Prep protein extraction solution (INtRON Biotechnology, Gyeonnggi-do, Korea) $48 \mathrm{~h}$ after transfection. An equal amount of proteins was resolved by $8 \%$ SDS-PAGE gels (Laemmli, 1970). The primary antibodies used for the analysis were mouse anti-EZH2 (1:1,000; BD Biosciences, San Jose, CA, USA) and mouse anti$\beta$-actin antibodies (1:2,000; Santa Cruz Biotechnology, Inc., Santa Cruz, CA, USA).

Chromatin immunoprecipitation assay (ChIP). ChIP was carried out using a kit purchased from Upstate Biotechnology (Charlottesville, VA, USA) according to the protocol recommended by the manufacturer. The trimethyl H3 (Lys-27) chip grade antibody was purchased from Abcam. The CDH1 gene was amplified by PCR from the immunoprecipitated chromatin using the following primers: sense, 5'-TAGAGGGTCACCGCGTCTAT-3' and antisense, 5'-TCACAGGTGCTTTGCAGTTC-3'. The PCR product was resolved on a $2 \%$ agarose gel and visualized using ethidium bromide staining and UV illumination.

Invasion assay. Invasion assays were performed in triplicate using 48-well microchemotaxis chambers with $8-\mu \mathrm{m}$ pore membranes (Millipore, Bedford, MA, USA) pre-coated with $10 \mu \mathrm{g} / \mathrm{ml}$ Matrigel (BD Bioscience). The cells were plated in 6-well plates and treated with $5 \mu \mathrm{M} \mathrm{5}$-azacytidine for $48 \mathrm{~h}$. The bottom chamber was filled with $1 \%$ penicillin/ RPMI-1640, and the cells were then placed in the upper chamber. A549 cells containing $5 \mu \mathrm{M}$ 5'-azacytidine were incubated at $37^{\circ} \mathrm{C}$ for $24 \mathrm{~h}$. The membranes were fixed and stained with Diff-Quik reagents (Dade Behring, Inc., Deerfield, IL, USA).

Statistical analysis. Statistical differences in the expression of miRNAs in cancer and normal lung tissues were analyzed using a non-parametric test (Mann-Whitney U test). Statistical differences in the expression of miRNAs in cell lines relative to the control were analyzed using the Student's t-test. Statistical significance of correlations between the global methylation of $\mathrm{H} 3 \mathrm{~K} 27$ and lung cancer invasion were analyzed by the Fisher extraction test. Statistical analysis was performed using SPSS 12.0 computer software (SPSS Inc., Chicago, IL, USA). A p-value $<0.05$ was considered to be statistically significant.

\section{Results}

miR-101 and EZH2 are inversely expressed in lung cancer tissue samples. To analyze the expression of miR-101 and EZH2 in lung cancer, qRT-PCR analysis for miRNAs was performed with 17 matched normal lung and lung cancer tissues. miR-101 exhibited decreased expression in the lung cancer tissues compared to the normal lung tissues $(\mathrm{p}<0.001)$. In contrast, EZH2 exhibited increased expression in the lung cancer tissues compared to the normal lung tissues $(p<0.001$; Fig. 1A). All cases of the 17 matched normal lung and lung cancer tissues exhibited a negative correlation (Fig. 1B). 
$\mathbf{A}$
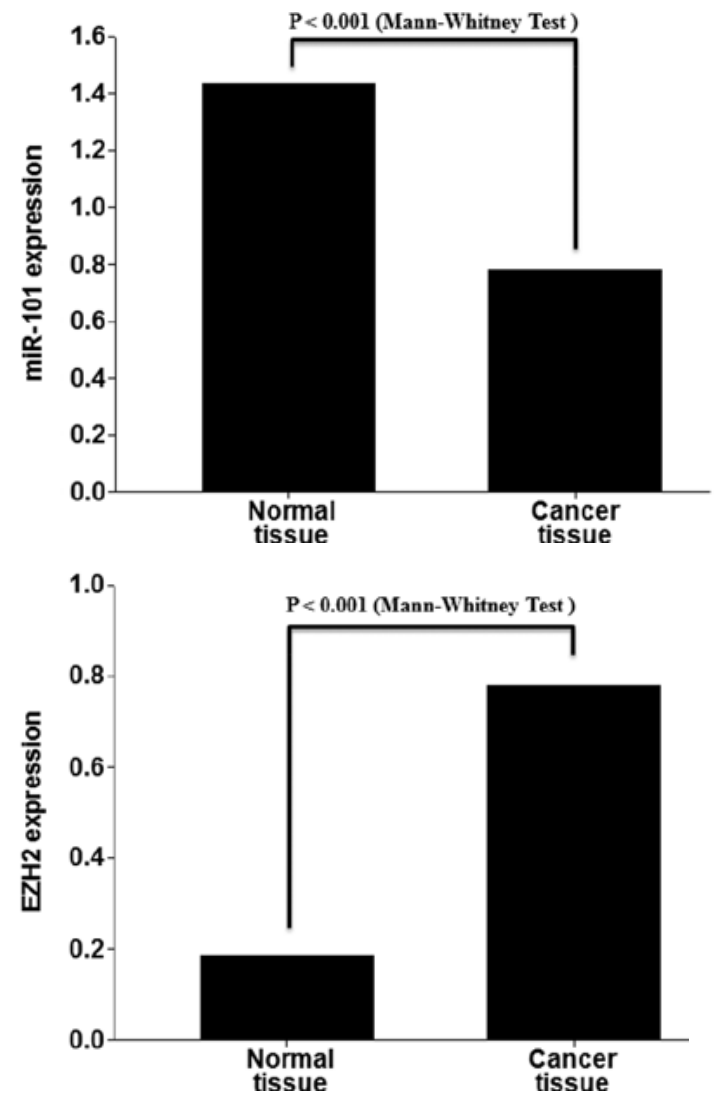

B

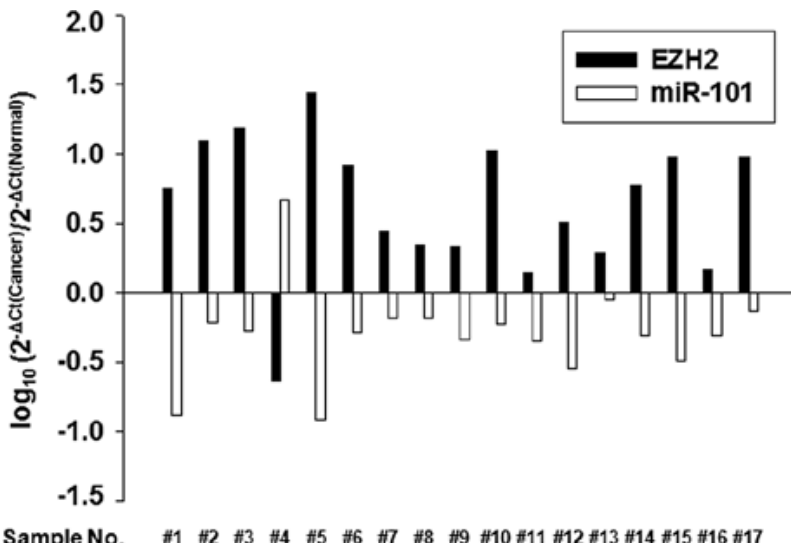

Figure 1. miR-101 and EZH2 are expressed in an opposite trend in lung cancer tissue samples. (A) Real-time RT-PCR analysis of mature miR-101 and EZH2 expression in lung cancer and normal lung tissues obtained from the same patients. (B) Quantitative real-time RT-PCR analysis of miR-101 and $\mathrm{EZH} 2$ in lung cancer tissues and matched normal tissues.

Global methylation of $H 3 K 27$ was highly expressed in the metastatic lesions. To determine the association of trimethylation of H3K27 (H3K27me3) with lung invasion, H3K27me3 was examined by IHC in 26 early lung cancer tissues without lymph node metastasis and 8 pleural metastatic tissues. H3K27me3 expression was significantly higher in the pleural metastatic lesions $(87.5 \%)$ than in the early lung cancer lesions $(42 \%)$ (p=0.042; Fig. 2).

EZH2, CDH1 and MMP-2 are regulated by miR-101 in lung cancer cells. To determine whether or not overexpression of miR-101 induces down-regulation of EZH2, qRT-PCR and Western blotting were performed in A549, H522 and H1438

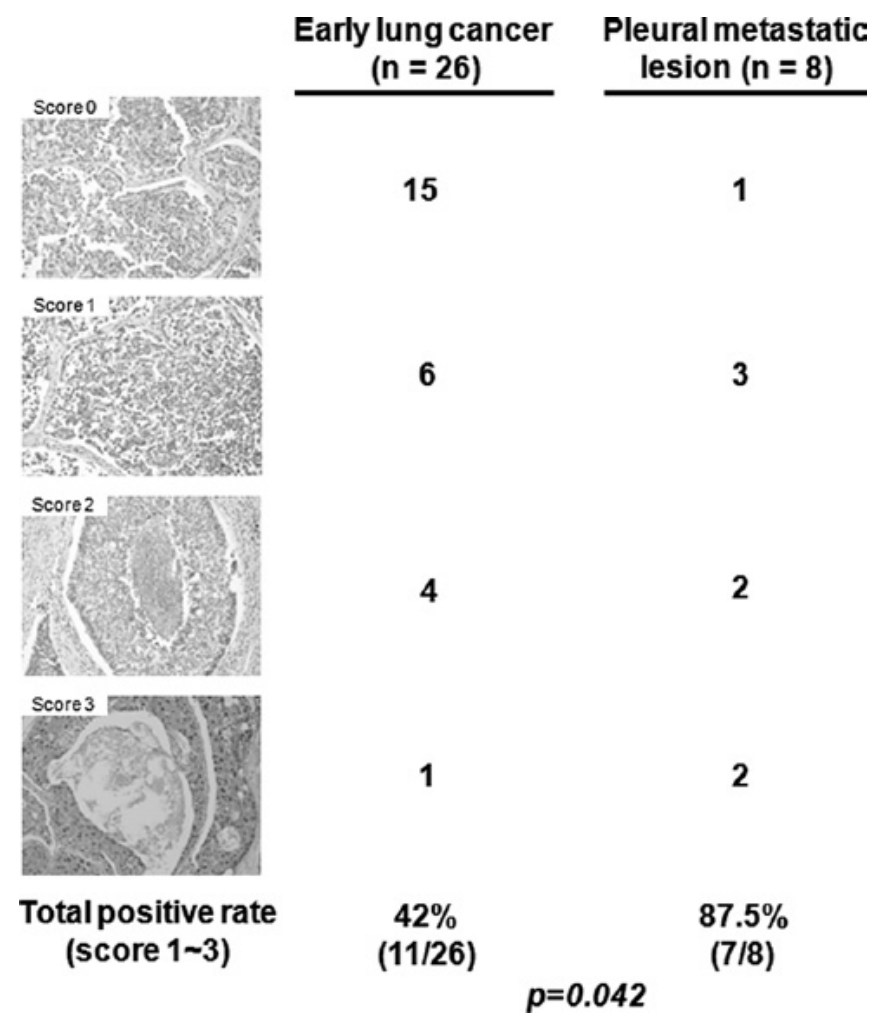

Figure 2. Global methylation of H3K27 is highly expressed in metastatic lesions. Histone $\mathrm{H} 3$ trimethyl K27 expression in lung cancer tissues was detected using the H3K27me3 antibody. Original magnification, x200.

cells transfected with the miR (negative control) or miR-101 precursor. Overexpression of miR-101 induced a marked reduction in EZH2 mRNA and EZH2 protein (Fig. 3A). To explore the possible regulation of invasion-related genes by miR-101 in lung cancer cells, the effect of miR-101 on the expression of CDH1 and MMP-2 in H1438 cells was analyzed. The overexpression of miR-101 had an inhibitory effect on the expression of the MMP-2 gene. In contrast, the expression of CDH1 was increased by overexpression of miR-101 (Fig. 3B). Next, the trimethylation of $\mathrm{H} 3 \mathrm{~K} 27$ (H3K27me3) of the $\mathrm{CDH}$ promoter was examined using a ChIP assay. A reduction was found in the trimethyl H3K27 histone mark at the promoter of $\mathrm{CDH} 1$ in the cells transfected with the miR-101 precursor (Fig. 3C).

miR-101 functions as an inhibitor of invasion in lung cancer. To test whether or not miR-101 causes inhibition of invasion, a cell invasion assay was performed in A549 lung cancer cells. miR-101 inhibited the invasiveness of A549 cells ( $<<0.005$; Fig. 4).

\section{Discussion}

Epigenetic alteration of genes is one of the central mechanisms involved in the development and progression of cancer, and includes hypermethylation of the $\mathrm{CpG}$ island on promoters and epigenetic modification states of histone. The global histone modification level predicts prognosis in various types of cancers (12-14). EZH2, which plays a part in the trimethylation of $\mathrm{H} 3 \mathrm{~K} 27$, leads to the silencing of tumor-suppressor genes. EZH2 is frequently overexpressed in various cancers, including prostate and breast cancer, and is associated with aggressive forms of these cancers $(15,16)$. In the present study, 


\section{Real-time RT-PCR}
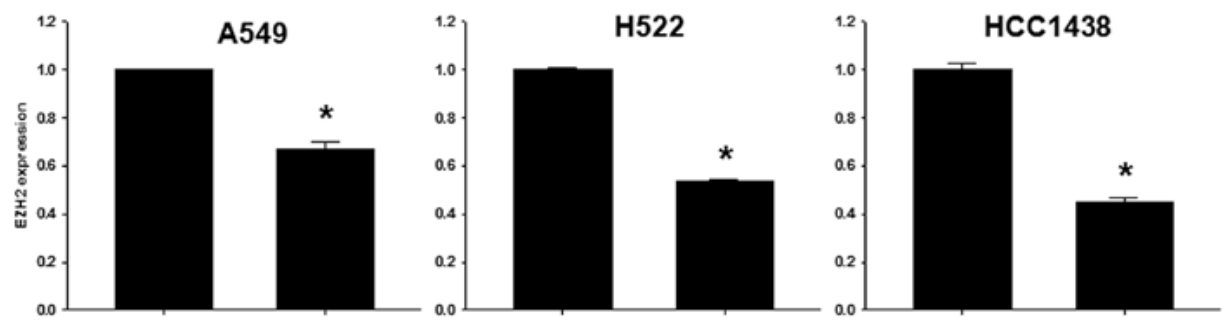

\section{Immuno Blot}
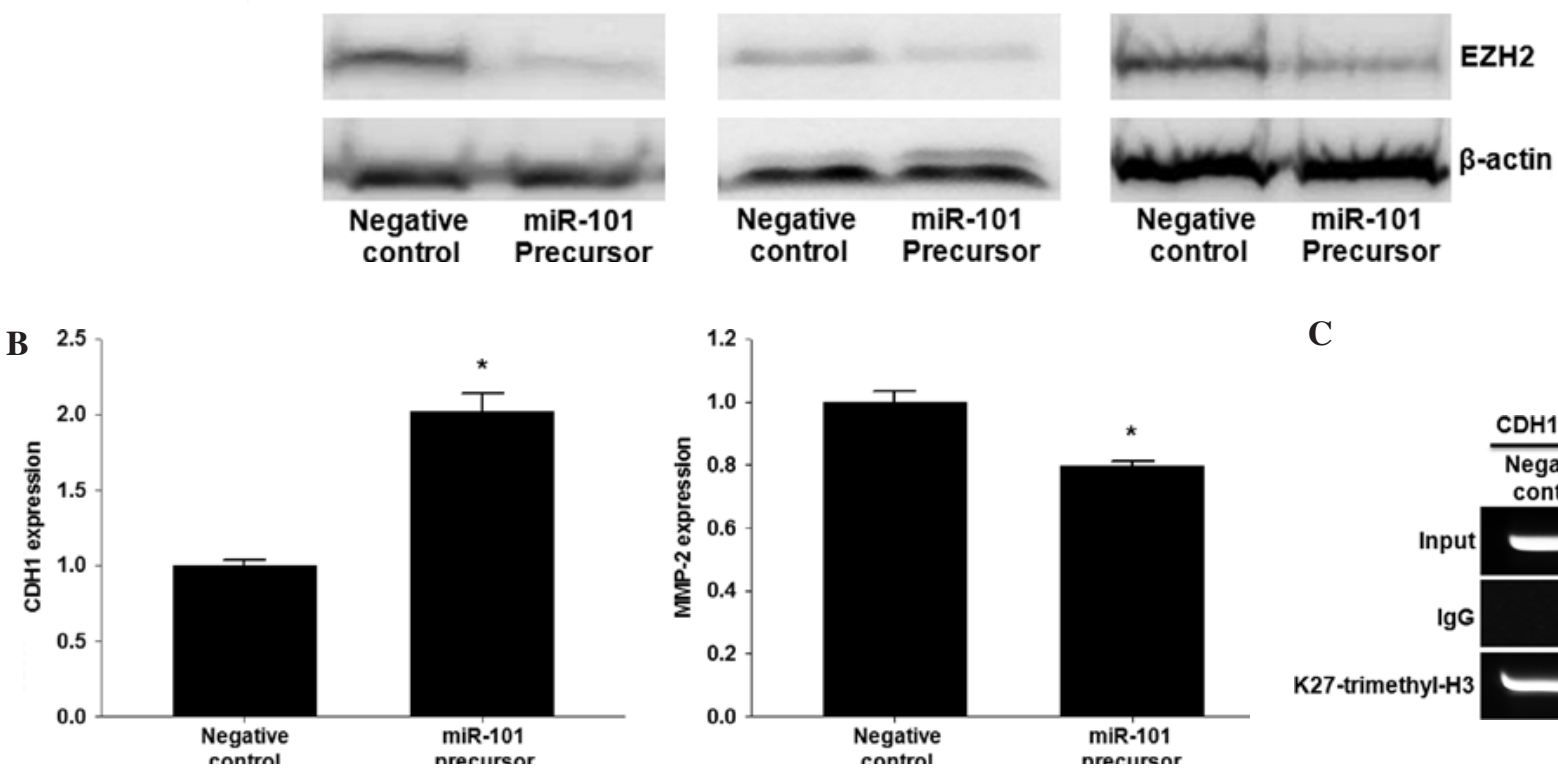

C (B) Effect of miR-101 on CDH1 and MMP-2 expression in lung cancer cells (HCC1438). (C) Chromatin immunoprecipitation (ChIP) assay of the trimethyl H3K27 histone mark after miR-101 precursor transfection was performed in A549 cells. ChIP was performed to test H3K27 trimethylation at the promoter of CDH1. Results show the means \pm standard deviation (SD) of three independent experiments performed in triplicate. ${ }^{*} \mathrm{p}<0.05$ vs. control.

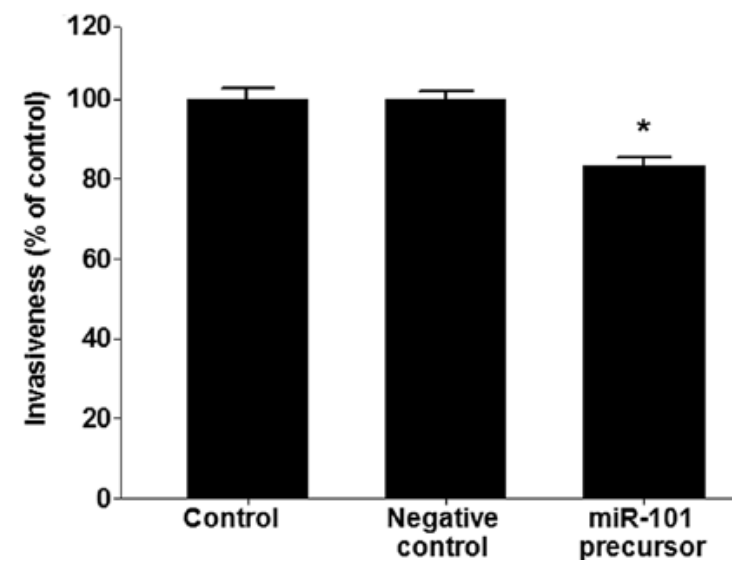

Figure 4. miR-101 functions as an inhibitor of invasion in lung cancer. A549 cells were transfected with miR-101 and negative controls. Results show the means \pm standard deviation (SD) of three independent experiments performed in triplicate. " $\mathrm{p}<0.05$ vs. control.

expression of $\mathrm{H} 3 \mathrm{~K} 27 \mathrm{me} 3$ in the metastatic lung cancer tissues was higher than in early lung cancer. This finding suggests that EZH2 plays a role in the progression of lung cancer.

Invasion is the most lethal feature of cancer. The initial stages of tumor invasion are characterized by the disrup- tion of cell-cell adhesion. CDH1 (E-cadherin) is involved in epithelial cell-cell communication and functions as a metastasis suppressor molecule $(17,18)$. The cytoplasmic tails of cadherins bind to several cytoplasmic proteins, most notably $\beta$-catenin, a transcriptional cofactor in Wnt signaling (19). Reduced E-cadherin expression is inversely correlated with lymph node stage and tumor differentiation in lung cancer (20). Moreover, reduced expression of E-cadherin is associated with the prognosis of patients with non-small cell lung cancer $(21,22)$. The process of invasion also requires a dynamic process, such as proteolysis. The degradation of extracellular membranes, which includes the basement membrane, is an early event in proteolysis. A subgroup of proteases, the matrix metalloproteinases (MMPs), a family of zinc- and calcium-dependent proteolytic enzymes, are essential for the breakdown of the basement membrane for metastatic spread $(23,24)$. In particular, MMP-2 interacts with integrin $\alpha \mathrm{V} \beta 3$ and increases VEGF expression through PI3K/AKT signaling in A549 lung cancer cells (25). Increased expression of MMP-2 promotes cell invasion and is a predictive biomarker for poor survival in non-small cell lung cancer (26-28).

$\mathrm{EZH} 2$ is known to play a role in the regulation of $\mathrm{CDH} 1$ and MMP-2. Overexpression of EZH2 induces silencing of CDH1 via trimethylation of $\mathrm{H} 3 \mathrm{~K} 27$ in the $\mathrm{CDH} 1$ promoter. Notably, a 
previous study showed that the expression of CDH1 and MMP-2 is inversely correlated (29) and $\mathrm{CDH} 1$ regulates the expression of MMP-2 $(30,31)$. The transition from a non-invasive phenotype to an invasive phenotype requires reduced $\mathrm{CDH} 1$ and overexpression of MMPs $(29,31)$. Our data suggest that EZH2 functions as the invasion mediator in lung cancer. Currently, miR-101 is considered a tumor-suppressive miRNA and has been reported to suppress cell proliferative and colony forming ability through EZH2 regulation in endometrial, bladder, liver, breast, prostate and gastric cancer (10,32-34). miR-101 has multiple predicted targets, such as Cox-2, Mcl-1 and FOS. These genes have been confirmed as targets of miR-101 by the luciferase reporter assay $(32,33,35)$. This evidence suggests that miR-101 modulates multiple steps, such as proliferation, adhesion and migration of tumor cells, in lung cancer.

In the present study, miR-101 was underexpressed and EZH2 was overexpressed in lung cancer. Substantial EZH2 suppression by miR-101 was observed at the mRNA and protein levels in lung cancer tissue samples. Moreover, overexpression of miR-101 in a lung cancer cell line altered the expression of CDH1 and MMP-2. Targeting tumor invasion and metastasis is an attractive strategy for the treatment of lung cancer. Therefore, these findings serve as an incentive for the further investigation of miR-101 as a therapeutic tool for lung cancer.

\section{Acknowledgements}

This study was supported by the National Research Foundation of Korea Grant funded by the Korean Government (NRF2009-0072810).

\section{References}

1. Ambros V: The functions of animal microRNAs. Nature 431: 350-355, 2004.

2. Kim VN and Nam JW: Genomics of microRNA. Trends Genet 22: 165-173, 2006.

3. Lim LP, Lau NC, Garrett-Engele P, et al: Microarray analysis shows that some microRNAs downregulate large numbers of target mRNAs. Nature 433: 769-773, 2005.

4. Bentwich I, Avniel A, Karov Y, et al: Identification of hundreds of conserved and nonconserved human microRNAs. Nat Genet 37: 766-770, 2005

5. Berezikov E, Guryev V, van de Belt J, Wienholds E, Plasterk RHA and Cuppen E: Phylogenetic shadowing and computational identification of human microRNA genes. Cell 120: 21-24, 2005.

6. Cheng AM, Byrom MW, Shelton J and Ford LP: Antisense inhibition of human miRNAs and indications for an involvement of miRNA in cell growth and apoptosis. Nucleic Acids Res 33: 1290-1297, 2005.

7. Xu P, Guo M and Hay BA: MicroRNAs and the regulation of cell death. Trends Genet 20: 617-624, 2004.

8. Karp X and Ambros V: Encountering microRNAs in cell fate signaling. Science 310: 1288-1289, 2005.

9. Chen C-Z, Li L, Lodish HF and Bartel DP: MicroRNAs modulate hematopoietic lineage differentiation. Science 303: 83-86, 2004.

10. Varambally S, Cao Q, Mani R-S, et al: Genomic loss of microRNA-101 leads to overexpression of histone methyltransferase EZH2 in cancer. Science 322: 1695-1699, 2008.

11. Friedman JM, Liang G, Liu C-C, et al: The putative tumor suppressor microRNA-101 modulates the cancer epigenome by repressing the polycomb group protein EZH2. Cancer Res 69: 2623-2629, 2009.

12. Anglim PP, Alonzo TA and Laird-Offringa IA: DNA methylation-based biomarkers for early detection of non-small cell lung cancer: an update. Mol Cancer 7: 81, 2008.

13. Barlesi F, Giaccone G, Gallegos-Ruiz MI, et al: Global histone modifications predict prognosis of resected non small-cell lung cancer. J Clin Oncol 25: 4358-4364, 2007.
14. Ellinger J, Kahl P, von der Gathen J, et al: Global levels of histone modifications predict prostate cancer recurrence. Prostate 70 : 61-69, 2010.

15. Varambally S, Dhanasekaran SM, Zhou M, et al: The polycomb group protein EZH2 is involved in progression of prostate cancer. Nature 419: 624-629, 2002.

16. Collett K, Eide GE, Arnes J, et al: Expression of enhancer of zeste homologue 2 is significantly associated with increased tumor cell proliferation and is a marker of aggressive breast cancer. Clin Cancer Res 12: 1168-1174, 2006.

17. Vleminckx K, Vakaet L, Mareel M, Fiers W and van Roy F: Genetic manipulation of E-cadherin expression by epithelial tumor cells reveals an invasion suppressor role. Cell 66: 107-119, 1991.

18. Semb H and Christofori G: The tumor-suppressor function of E-cadherin. Am J Hum Genet 63: 1588-1593, 1998.

19. Halbleib JM and Nelson WJ: Cadherins in development: cell adhesion, sorting, and tissue morphogenesis. Genes Dev 20: 3199-3214, 2006

20. Sulzer MA, Leers MP, van Noord JA, Bollen EC and Theunissen PH: Reduced E-cadherin expression is associated with increased lymph node metastasis and unfavorable prognosis in non-small cell lung cancer. Am J Respir Crit Care Med 157: 1319-1323, 1998.

21. Liu D, Huang C, Kameyama K, et al: E-cadherin expression associated with differentiation and prognosis in patients with non-small cell lung cancer. Ann Thorac Surg 71: 949-955, 2001.

22. Huang C, Liu D, Masuya D, et al: Clinical application of biological markers for treatments of resectable non-small cell lung cancers. Br J Cancer 92: 1231-1239, 2005.

23. Liotta LA, Tryggvason K, Garbisa S, Hart I, Foltz CM and Shafie S: Metastatic potential correlates with enzymatic degradation of basement membrane collagen. Nature 284: 67-68, 1980.

24. Nelson AR, Fingleton B, Rothenberg ML and Matrisian LM: Matrix metalloproteinases: biologic activity and clinical implications. J Clin Oncol 18: 1135, 2000.

25. Chetty C, Lakka SS, Bhoopathi P and Rao JS: MMP-2 alters VEGF expression via $\alpha \mathrm{V} \beta 3$ integrin-mediated PI3K/AKT signaling in A549 lung cancer cells. Int J Cancer 127: 1081-1095, 2010.

26. Leinonen T, Pirinen R, Bohm J, Johansson R and Kosma VM: Increased expression of matrix metalloproteinase-2 (MMP-2) predicts tumour recurrence and unfavourable outcome in non-small cell lung cancer. Histol Histopathol 23: 693-700, 2008.

27. Kim S-H, Choi HY, Lee J, et al: Elevated activities of MMP-2 in the non-tumorous lung tissues of curatively resected stage I NSCLC patients are associated with tumor recurrence and a poor survival. J Surg Oncol 95: 337-346, 2007.

28. Hung W-C, Tseng W-L, Shiea J and Chang H-C: Skp2 overexpression increases the expression of MMP-2 and MMP-9 and invasion of lung cancer cells. Cancer Lett 288: 156-161, 2010.

29. Polette M, Gilles C, de Bentzmann S, Gruenert D, Tournier J-M and Birembaut P: Association of fibroblastoid features with the invasive phenotype in human bronchial cancer cell lines. Clin Exp Metastasis 16: 105-112, 1998.

30. Miyaki M, Tanaka K, Kikuchi-Yanoshita R, Muraoka M, Konishi $\mathrm{M}$ and Takeichi M: Increased cell-substratum adhesion, and decreased gelatinase secretion and cell growth, induced by E-cadherin transfection of human colon carcinoma cells. Oncogene 11: 2547-2552, 1995.

31. Nawrocki-Raby B, Gilles C,Polette M, et al: E-cadherin mediates MMP down-regulation in highly invasive bronchial tumor cells. Am J Pathol 163: 653-661, 2003.

32. Su H, Yang J-R, Xu T, et al: MicroRNA-101, down-regulated in hepatocellular carcinoma, promotes apoptosis and suppresses tumorigenicity. Cancer Res 69: 1135-1142, 2009.

33. Li S, Fu H, Wang Y, et al: MicroRNA-101 regulates expression of the v-fos FBJ murine osteosarcoma viral oncogene homolog (FOS) oncogene in human hepatocellular carcinoma. Hepatology 49: 1194-1202, 2009.

34. Wang H-J, Ruan H-J, He X-J, et al: MicroRNA-101 is downregulated in gastric cancer and involved in cell migration and invasion. Eur J Cancer 46: 2295-2303, 2010.

35. Strillacci A, Griffoni C, Sansone P, et al: MiR-101 downregulation is involved in cyclooxygenase-2 overexpression in human colon cancer cells. Exp Cell Res 315: 1439-1447, 2009. 